\title{
Is complete mesocolic excision oncologically superior to conventional surgery for colon cancer? A retrospective comparative study
}

\section{Christos Agalianos ${ }^{a}$, Nikolaos Gouvas ${ }^{b}$, Christos Dervenisc, John Tsiaoussis ${ }^{d}, G^{c}$ George Theodoropoulos ${ }^{\mathrm{e}}$, Demetrios Theodorou' ${ }^{e}$, George Zografos ${ }^{e}$, Evaghelos Xynos ${ }^{f}$}

Athens Naval \& Veterans Hospital, Athens; Worcestershire Acute Hospitals Trust, UK; Konstantopouleio Hospital of Athens; School of Medicine, University of Crete; Hippokration Hospital, University of Athens; Creta Interclinic Hospital of Herakleion, Crete, Greece

\section{Abstract}

${ }^{a}$ Department of General Surgery, Athens Naval \& Veterans Hospital, Athens, Greece (Christos Agalianos); ${ }^{b}$ Department of Surgery, Worcestershire Acute Hospitals Trust, UK (Nikolaos Gouvas); 'Department of Surgery, "Konstantopouleio" Hospital of Athens (Christos Dervenis); dDepartment of Anatomy \& Embryology, School of Medicine, University of Crete (John Tsiaoussis); ${ }^{e} 1^{\text {st }}$ Propedeutic Surgical Department, Hippokration Hospital, University of Athens (George Theodoropoulos, Demetrios Theodorou, George Zografos); ${ }^{\mathrm{f}}$ Creta Interclinic Hospital of Heraklion, Crete (Evangelos Xynos)

Conflict of Interest: None

Correspondence to: Christos Agalianos, Department of General Surgery, Athens Naval \& Veterans Hospital, 70 Dinokratous Str., 11521 Athens, Greece, e-mail address: xagali@gmail.com

Received 16 May 2017; accepted 24 August 2017; published online 4 October 2017

DOI: https://doi.org/10.20524/aog.2017.0197

\section{Introduction}

Total mesorectal excision for rectal cancer has resulted in excellent oncological outcomes, achieving lower rates of local recurrence and higher rates of both disease-free and overall survival rates, because all possible routes of spread are removed in a package, through dissection along well-defined embryological planes [1-3]. Hohenberger et al [4] thought that the same principles could be also applied to colon cancer surgery, by introducing the concept of complete mesocolic excision (CME). CME involves sharp dissection of the mesocolon along the embryological planes and high ligation and division of the vessels supplying blood to the colonic segment to be removed. By this technique, the removed colonic specimen should be covered by an intact mesocolic fascia and peritoneum, which, as a package, contains the 
tumor-bearing colonic segment and the mesocolon, with draining lymphatics, lymph nodes and possible tumor microdeposits.

Many studies document clearly that CME results in more tissue being obtained in terms of the number of harvested lymph nodes, the distance from high tie to tumor and the surface of removed mesocolon, compared to conventional surgery [4-11]. However, the concept of CME remains controversial, with low uptake worldwide [6,12], mostly because the improved morphological features attributed to CME fail to translate into improved oncological outcomes. Observational studies $[4,8,11]$ show that CME is associated with higher overall survival rate compared to historical series of patients after conventional surgery. More recently, there are comparative, population based studies demonstrating higher overall $[12,13]$ and disease-free [12-14] survival rates after CME compared to conventional surgery, in particular for stage I and II right colon cancers.

The present comparative study aims to add further information based on the aforementioned principles; namely, management of patients under the control of multidisciplinary teams that follow the same guidelines for treatment and macroscopic, microscopic and histopathological assessment of the resected specimen based on the same rules.

\section{Patients and methods}

\section{Patients}

Adult patients (18 years old or older) from two surgical units were included. All colon cancer patients undergoing surgery were registered in a prospective electronic database. All patients who underwent surgery for colon cancer (lower border of tumor no less than $15 \mathrm{~cm}$ from the anal verge as measured by colonoscopy) from October 2006 to December 2011 were included in this study. All patients had histologically-proven adenocarcinoma of the colon and were staged by computed tomography (CT) scans. Exclusion criteria included synchronous metastatic disease and patients undergoing emergency surgery. All CME operations were performed or supervised by one specially trained surgeon (EX) in colorectal surgery. All other surgeons in the two units performed conventional colectomies, but all of them were equally experienced general surgeons with a special interest in colorectal surgery. All operations were performed by the open approach. All patients in the two units were enrolled postoperatively and managed within an enhanced recovery protocol. All CME specimens were freshly photographed and evaluated macroscopically and morphometrically using special software (Adobe Photoshop CS6), using the technique and standard definitions of the pathologists in Leeds, UK [11]. Two specially trained and experienced pathologists (KP, MK) evaluated all specimens from both groups histologically.

\section{Surgical techniques}

In left colectomy the procedure commenced by dissecting laterally to medially the embryological plane between the visceral and the parietal layer of Toldt's fascia. The left colon was mobilized in this way from the left paracolic gutter. The inferior mesenteric artery was then ligated and transected approximately $1 \mathrm{~cm}$ after its take-off from the aorta, so as to preserve the hypogastric nerve plexus, after which the inferior mesenteric vein was ligated and transected immediately below the inferior border of the pancreas. Dissection extended caudally over the promontory and to the upper "holy plane" and superiorly to the entry of the lesser epiploic sac, after division of the root of the left transverse mesocolon at the lower border of the pancreas. The sigmoid colon was excised in all cases, and a descending colon to mid or upper rectum anastomosis was fashioned using a circular stapler.

In right colectomy, the procedure started with a lateralto-medial dissection in the plane defined between the two layers of Toldt's fascia to the anterior surface of the superior mesenteric vessels, below the third portion of the duodenum. In cases with a carcinoma located in the cecum or the proximal ascending colon, the ileo-colic vessels were taken from their origin at the superior mesenteric vessels and the right branches of the middle colic vessels at their take-off from the main trunks. When the carcinoma was located in the hepatic flexure or the proximal $(15 \mathrm{~cm})$ transverse colon, the middle colic vessels were dissected, ligated and transected at the origin from the superior mesenteric vessels. Then, the mesentery of the colon to be excised was divided, as were the distal ileum and the transverse colon. The anastomosis was fashioned either with sutures or with the use of linear stapling devices. The omentum related to the resected specimen was excised en bloc. In addition, for carcinomas of the hepatic flexure and proximal transverse colon, the hypo-pyloric and gastro-epiploic lymph nodes were removed en bloc with the specimen.

Non-CME operations also involved the mobilization of the colon by developing the embryological plane between the visceral and the parietal layers of Toldt's fascia. However, the surgeons did not systematically try to preserve the planes intact. Furthermore, the level of the high tie was not standardized according to a fixed anatomical structure, which resulted in leaving behind a considerable amount of lymph tissue along the remaining ligated arterial stump.

\section{Follow up}

Chemotherapy was offered to all stage III patients by the Multidisciplinary Team as standard treatment in the form of mFOLFOX6. Stage II patients received adjuvant chemo only if histological and/or clinical adverse features were present, such as invasion of blood vessels or lymphatics, poor differentiation, perineural invasion, number of retrieved lymph nodes below 12, pT4 tumors and tumors causing obstruction. Furthermore, every stage II patient after 2009 without the above adverse pathologic features was tested for microsatellite 
instability; if the tumor displayed microsatellite stability the patient was offered adjuvant chemotherapy. All patients were followed according to the international guidelines for patients with colon cancer. The date of last follow up was considered the date of the last chest and abdomen CT scan or the date of any additional test used to identify possible recurrence.

\section{Statistical analysis}

Nominal variables are presented using frequencies and percentages, whereas continuous variables are presented as median and interquartile range (normality was tested and did not hold).

The $\mathrm{x}^{2}$ test for independence was used to evaluate associations between the procedure group and patients' characteristics. Kaplan-Meier survival analysis was performed in order to evaluate differences in the disease-free survival time and overall survival between the two procedure groups with the use of a log-rank test. Lastly, Cox regression was performed in order to identify factors independently related to the patients' disease-free and overall survival time. The factors entered into the model were those that were statistically significant in the univariate log-rank tests. Results are presented as hazard ratios and $95 \%$ confidence intervals (CI).

The criterion of statistical significance was $\alpha=5 \%$. All statistical analyses were performed using of Stata SE version 11.1 (StataCorp. 2009, Texas, USA).

\section{Results}

\section{Demographics, intraoperative and postoperative outcomes}

Two hundred ninety patients were eligible for analysis. Table 1 shows the patients' demographics, preoperative tumor characteristics and types of operation performed. Apart from age, no other statistically significant difference was identified between the two groups.

There were no differences between the two groups as far as intraoperative events are concerned. Overall intraoperative complications $(\mathrm{P}=0.652)$, and in particular intraoperative bleeding $(\mathrm{P}=0.156)$ and failure of anastomosis $(\mathrm{P}=0.081)$, showed no difference between the two groups. Thirtyday mortality rates also did not differ between the groups. The CME group displayed significantly higher rates of postoperative morbidity $(\mathrm{P}<0.001)$, and when morbidity was further analyzed the CME group had higher rates of chest infection ( 31 vs. 0 patients, $\mathrm{P}<0.001$ ) and postoperative ileus ( 31 vs. 7 patients, $\mathrm{P}<0.001$ ). Furthermore, there were significantly more readmissions in the CME group (6 vs. 0 patients, $\mathrm{P}=0.013$ ) whereas reoperation rates, bleeding, leak rates and postoperative ileus cases did not differ between the groups. Although there were more readmissions in the CME group, total hospital stay was significantly shorter when compared to the non-CME group (6 [4-8] vs. 7 [6-8] days, $\mathrm{P}<0.001)$. All readmissions $(\mathrm{n}=6)$ in the $\mathrm{CME}$ group were due to prolonged postoperative ileus.

\section{Histopathological and oncological outcomes}

Table 2 presents the association between the procedure groups and histopathological and oncological outcomes. The CME group had a significantly lower number of patients who were offered adjuvant chemotherapy $(\mathrm{P}=0.012)$, possibly because it included a significantly lower number of patients

Table 1 Patients and tumor characteristics in the two groups

\begin{tabular}{|c|c|c|c|}
\hline \multirow[t]{2}{*}{ Characteristics } & \multicolumn{2}{|c|}{ n (\%) } & \multirow[t]{2}{*}{ P-value } \\
\hline & $\begin{array}{c}\text { Non-CME } \\
n=145\end{array}$ & $\begin{array}{c}\text { CME } \\
\mathrm{n}=145\end{array}$ & \\
\hline Age & $72(65-78)^{*}$ & $68(58-75)^{*}$ & $<0.001$ \\
\hline \multicolumn{4}{|l|}{ Sex } \\
\hline Male & $79(54.48 \%)$ & $88(60.69 \%)$ & 0.285 \\
\hline Female & $66(45.52 \%)$ & $57(39.31 \%)$ & \\
\hline \multicolumn{4}{|l|}{ Tumor location } \\
\hline Cecum - ascending & $55(39.57 \%)$ & $42(29.17 \%)$ & 0.219 \\
\hline Hepatic flexure - transverse & $16(11.51 \%)$ & $25(17.36 \%)$ & \\
\hline Descending - upper sigmoid & $17(12.23 \%)$ & $22(15.28 \%)$ & \\
\hline Sigmoid - rectosigmoid & $51(36.69 \%)$ & $55(38.19 \%)$ & \\
\hline \multicolumn{4}{|l|}{ Localization } \\
\hline Right & $71(48.97 \%)$ & $68(46.90 \%)$ & 0.724 \\
\hline Left & $74(51.03 \%)$ & $77(53.10 \%)$ & \\
\hline
\end{tabular}

Table 2 Histopathological and oncological data

\begin{tabular}{|c|c|c|c|}
\hline \multirow[t]{2}{*}{ Variable } & \multicolumn{2}{|c|}{$\mathrm{n}(\%)$} & \multirow[t]{2}{*}{ P-value } \\
\hline & $\begin{array}{c}\text { Non-CME } \\
n=145\end{array}$ & $\begin{array}{c}\text { CME } \\
\mathrm{n}=145\end{array}$ & \\
\hline CME grading & & & - \\
\hline Mesocolic & - & 135 (93.1\%) & \\
\hline Intramesocolic & - & $10(6.9 \%)$ & \\
\hline Muscularis & - & $0(0 \%)$ & \\
\hline \multicolumn{4}{|l|}{ Differentiation } \\
\hline Low & $29(20.00 \%)$ & $38(26.210 \%)$ & 0.210 \\
\hline High & $116(80.00 \%)$ & $107(73.79 \%)$ & \\
\hline \multicolumn{4}{|l|}{$\begin{array}{l}\text { Adjuvant } \\
\text { Chemotherapy }\end{array}$} \\
\hline No & $66(46.48 \%)$ & $87(61.27 \%)$ & 0.012 \\
\hline Yes & 76 (53.53\%) & $55(38.73 \%)$ & \\
\hline
\end{tabular}


Table 2 (Contined)

\begin{tabular}{|c|c|c|c|}
\hline \multirow[t]{2}{*}{ Variable } & \multicolumn{2}{|c|}{ n (\%) } & \multirow[t]{2}{*}{ P-value } \\
\hline & $\begin{array}{c}\text { Non-CME } \\
n=145\end{array}$ & $\begin{array}{c}\text { CME } \\
\mathrm{n}=145\end{array}$ & \\
\hline \multicolumn{4}{|l|}{ T stage } \\
\hline T0 & $0(0.00 \%)$ & $1(0.69 \%)$ & $<0.001$ \\
\hline $\mathrm{T} 1$ & $6(4.14 \%)$ & $17(11.72 \%)$ & \\
\hline $\mathrm{T} 2$ & $11(7.59 \%)$ & $37(25.52 \%)$ & \\
\hline $\mathrm{T} 3$ & $104(71.72 \%)$ & $69(47.59 \%)$ & \\
\hline $\mathrm{T} 4$ & $21(14.48 \%)$ & $21(14.48 \%)$ & \\
\hline Tis & $3(2.07 \%)$ & $0(0.00 \%)$ & \\
\hline \multicolumn{4}{|l|}{$\mathrm{N}$ stage } \\
\hline No & $80(55.17 \%)$ & $101(69.66 \%)$ & 0.021 \\
\hline N1 & $40(27.59 \%)$ & $32(22.07 \%)$ & \\
\hline N2 & $25(17.24 \%)$ & $11(7.59 \%)$ & \\
\hline N3 & $0(0.00 \%)$ & $1(0.69 \%)$ & \\
\hline \multicolumn{4}{|l|}{ UICC stage } \\
\hline Stage I & $13(8.97 \%)$ & $48(33.10 \%)$ & $<0.001$ \\
\hline Stage II & $67(46.21 \%)$ & $51(35.17 \%)$ & \\
\hline Stage III & $65(44.83 \%)$ & $46(31.72 \%)$ & \\
\hline \multicolumn{4}{|l|}{ Local recurrence } \\
\hline No & $137(96.48 \%)$ & $136(94.44 \%)$ & 0.409 \\
\hline Yes & $5(3.52 \%)$ & $8(5.56 \%)$ & \\
\hline \multicolumn{4}{|l|}{ Metastasis } \\
\hline No & $118(83.10 \%)$ & $125(86.81 \%)$ & 0.380 \\
\hline Yes & $24(16.90 \%)$ & $19(13.19 \%)$ & \\
\hline \multicolumn{4}{|l|}{ Death } \\
\hline No & $109(75.69 \%)$ & $125(86.21 \%)$ & 0.023 \\
\hline \multirow[t]{2}{*}{ Yes } & $35(24.31 \%)$ & $20(13.79 \%)$ & \\
\hline & Median (IQR) & Median (IQR) & \\
\hline $\begin{array}{l}\text { Lymph nodes } \\
\text { retrieved }\end{array}$ & $18(14-24)$ & $27(19-37)$ & $<0.001$ \\
\hline Bowel length & $20(17-25)$ & $33(27-42)$ & $<0.001$ \\
\hline $\begin{array}{l}\text { Ileum length } \\
\text { (right only) }\end{array}$ & $5.5(4-8)$ & $6.5(4.75-7.25)$ & 0.943 \\
\hline $\begin{array}{l}\text { Tumor ligation } \\
\text { length }\end{array}$ & - & $14(12.5-16)$ & - \\
\hline $\begin{array}{l}\text { Bowel ligation } \\
\text { length }\end{array}$ & - & $10(8.5-12)$ & - \\
\hline $\begin{array}{l}\text { Mesocolon } \\
\text { surface }\end{array}$ & - & $171(133.5-218)$ & - \\
\hline
\end{tabular}

with more advanced stages of disease ( $\mathrm{T}, \mathrm{N}$ and UICC stage, $\mathrm{P}<0.001, \mathrm{P}=0.021$ and $\mathrm{P}<0.001$, respectively). Patients treated with non-CME had a lower percentage of stage I cancer (8.97\%) than patients undergoing CME (33.10\%), whereas

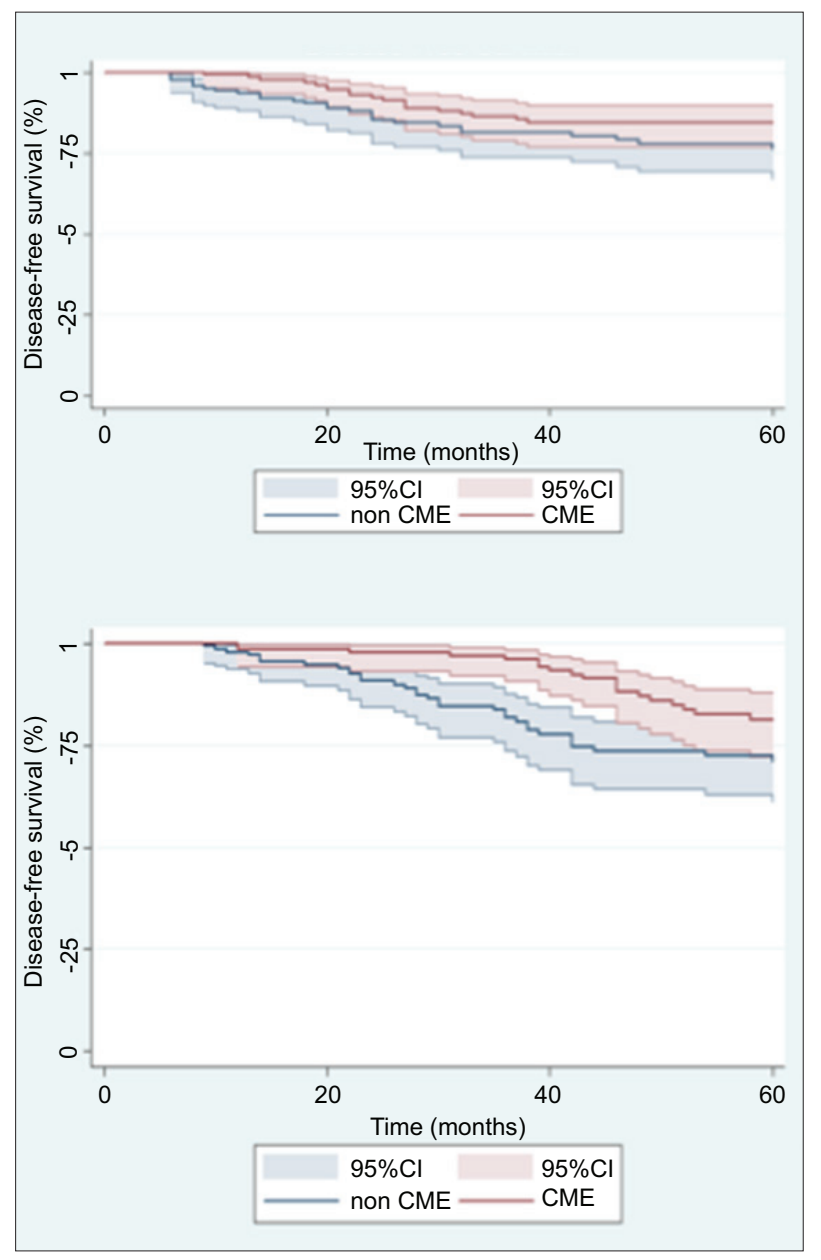

Figure 1 Survival curves by procedure group CME, complete mesocolic excision; CI, confidence interval

patients who had CME had a longer resected bowel (median 33 vs. $20 \mathrm{~cm}$ ) and more lymph nodes retrieved (median 27) than patients without CME (median 18).

\section{Survival analysis}

Five-year disease-free survival was $84.6 \%$ (95\%CI 76.9 89.9\%) after CME and 76.4\% (95\%CI 67.1-83.4\%) after nonCME (Fig. 1). In general, CME did not statistically significantly improve disease-free survival, but a longer disease-free survival time was observed for patients after CME than after non-CME (mean survival time 54.5 vs. 51.7 months, $\mathrm{P}=0.118$ ). With respect to overall survival, CME procedure improved the survival time significantly ( $\mathrm{P}=0.014$, Fig. 1). Patients with $\mathrm{CME}$ had longer overall survival times than those without $\mathrm{CME}$ (mean survival time 56.6 vs. 51.7 months). Five-year overall survival was $81.3 \%$ (95\%CI 72.1-87.7\%) after CME and 70.9\% (95\%CI 61.2-78.7\%) after non-CME.

Stratified survival analysis to explore the association between treatment groups and disease-free survival time in subgroups (Table 3 ) showed a statistically significant association 
Table 3 Stratified associations between treatment group and survival time

\begin{tabular}{|c|c|c|c|}
\hline \multirow[t]{2}{*}{ Associations } & Non-CME & CME & Log-rank \\
\hline & Mean (95\% CI) & Mean $(95 \%$ CI) & P-value \\
\hline \multicolumn{4}{|c|}{ Disease-free survival } \\
\hline \multicolumn{4}{|l|}{ By stage } \\
\hline Stage I & 60 & 60 & - \\
\hline Stage II & $56.5(53.6-59.8)$ & $58.2(55.7-60.6)$ & 0.347 \\
\hline Stage III & $45.4(40-50.7)$ & $44.7(39.1-50.4)$ & 0.874 \\
\hline \multicolumn{4}{|c|}{ By differentiation } \\
\hline Low & $50.1(42.5-57.8)$ & $48.2(42.7-53.8)$ & 0.853 \\
\hline High & $52.2(49-55.4)$ & $57.1(54.9-59.2)$ & 0.013 \\
\hline \multicolumn{4}{|l|}{ By localization } \\
\hline Right & $51.1(46.6-55.5)$ & $54.8(51.5-58.2)$ & 0.131 \\
\hline Left & $52.3(48.4-56.3)$ & $54.3(51.2-57.5)$ & 0.553 \\
\hline \multicolumn{4}{|c|}{ Overall survival } \\
\hline \multicolumn{4}{|l|}{ By stage } \\
\hline Stage I & 60 & 60 & - \\
\hline Stage II & $53.7(50.2-57.3)$ & $57.7(55.3-60.1)$ & 0.157 \\
\hline Stage III & $47.9(43.5-52.4)$ & $51.5(47.8-55.3)$ & 0.673 \\
\hline \multicolumn{4}{|c|}{ By differentiation } \\
\hline Low & $49.9(43.2-56.5)$ & $53.7(50.2-57)$ & 0.920 \\
\hline High & $52.2(49.2-55)$ & $57.9(56.4-59.6)$ & $<0.001$ \\
\hline \multicolumn{4}{|c|}{ By localization } \\
\hline Right & $51.2(47.2-55.2)$ & $55.7(52.8-58.6)$ & 0.108 \\
\hline Left & $52.2(48.6-55.7)$ & $57.4(55.9-58.9)$ & 0.071 \\
\hline
\end{tabular}

for patients that had high differentiation. Among those who had high differentiation, patients who had undergone a CME procedure had longer disease-free survival time than those undergoing non-CME. Stratification by stage did not show any statistically significant difference between groups (Table 3, Fig. 2). Likewise, there was an interaction between $\mathrm{CME}$ and differentiation, as well as adjuvant chemotherapy, for the overall survival time. Among patients who had highly differentiated tumors, it was found that the CME procedure resulted in a longer overall survival time compared to those who had a non-CME procedure (mean 57.9 vs. 52.2 months, $\mathrm{P}<0.001$ ). In terms of survival with reference to death from cancer, interaction was also observed between CME and differentiation $(\mathrm{P}=0.005)$.

Characteristics that could potentially affect the diseasefree survival or the overall survival were tested with the log-rank test. It was observed that disease-free and overall survival were statistically associated with differentiation and stage $(\mathrm{P}<0.05)$. Patients diagnosed with stage III disease and with low differentiation had shorter overall survival time and disease-free survival time, as expected (Fig. 3, 4). Patients with stage II disease did not have statistically different disease- free survival time from patients with stage I disease, but overall survival did differ $(\mathrm{P}=0.051$ for disease-free and $\mathrm{P}=0.003$ for overall survival). Moreover, age was also associated with overall survival $(\mathrm{P}=0.001)$. No significant difference in diseasefree or overall survival was detected for factors such as sex, localization, tumor location, approach, complications, number of lymph nodes retrieved, and hospital stay.

Multivariable Cox regression showed that CME is not an independent predictive factor for higher disease-free survival or overall survival (Table 4), since the hazard ratio was not statistically significant. Differentiation, adjuvant chemotherapy and age were shown to be statistically significant predictive factors for disease-free and overall survival rates $(\mathrm{P}<0.05)$. Stratified analysis by stage showed that there were no statistically significant factors associated with disease-free and overall survival rates for patients with stage II cancer. In contrast, for patients with stage III cancer, low differentiation was shown to be a statistically significant independent predictive factor for lower disease-free and overall survival rates.

\section{Discussion}

The concept of CME with central vascular ligation (CVL) has received very strong criticism. The initial criticism questioned the novelty of the technique. Many maintained that it was a concept already implemented by the majority of colorectal surgeons, in particular for tumors of the left colon $[15,16]$. In addition, the issue was raised of the reproducibility of the technique, because of the high level of surgical expertise required, along with concerns about higher rates of morbidity and intraoperative life-threatening adverse events. Many experienced and skillful colorectal surgeons claimed that some steps of CME with CVL, as described by Hohenberger et al [4], were technically very demanding, some were unnecessary, and because of that the technique was rendered very difficult to teach and to reproduce [13,17-19].

Our study failed to identify CME as an independent predictive factor for better disease-free and overall survival rates when compared with conventional colectomy, even when the disease was stratified by stage. Although survival analysis of the entire groups revealed a statistically significant difference favoring the CME group only as far as overall survival was concerned, when the cohort was stratified by several confounders, such as age, adjuvant chemotherapy, tumor differentiation and CME, the only significant independent predictive factor for both disease-free and overall survival rates, especially for UICC stage III patients, was high differentiation of tumors, which was associated with a significantly better disease-free and overall survival rates in the CME group.

Because there was a significant difference between the percentages of different stages of the disease between the two groups, all analyses were stratified by stage in order to confront this important source of bias. Nevertheless, CME failed to be identified as an independent predictive factor for disease-free and overall survival rates. This finding strongly contradicts many studies in the current literature $[4,8,11-14,20]$. The most 


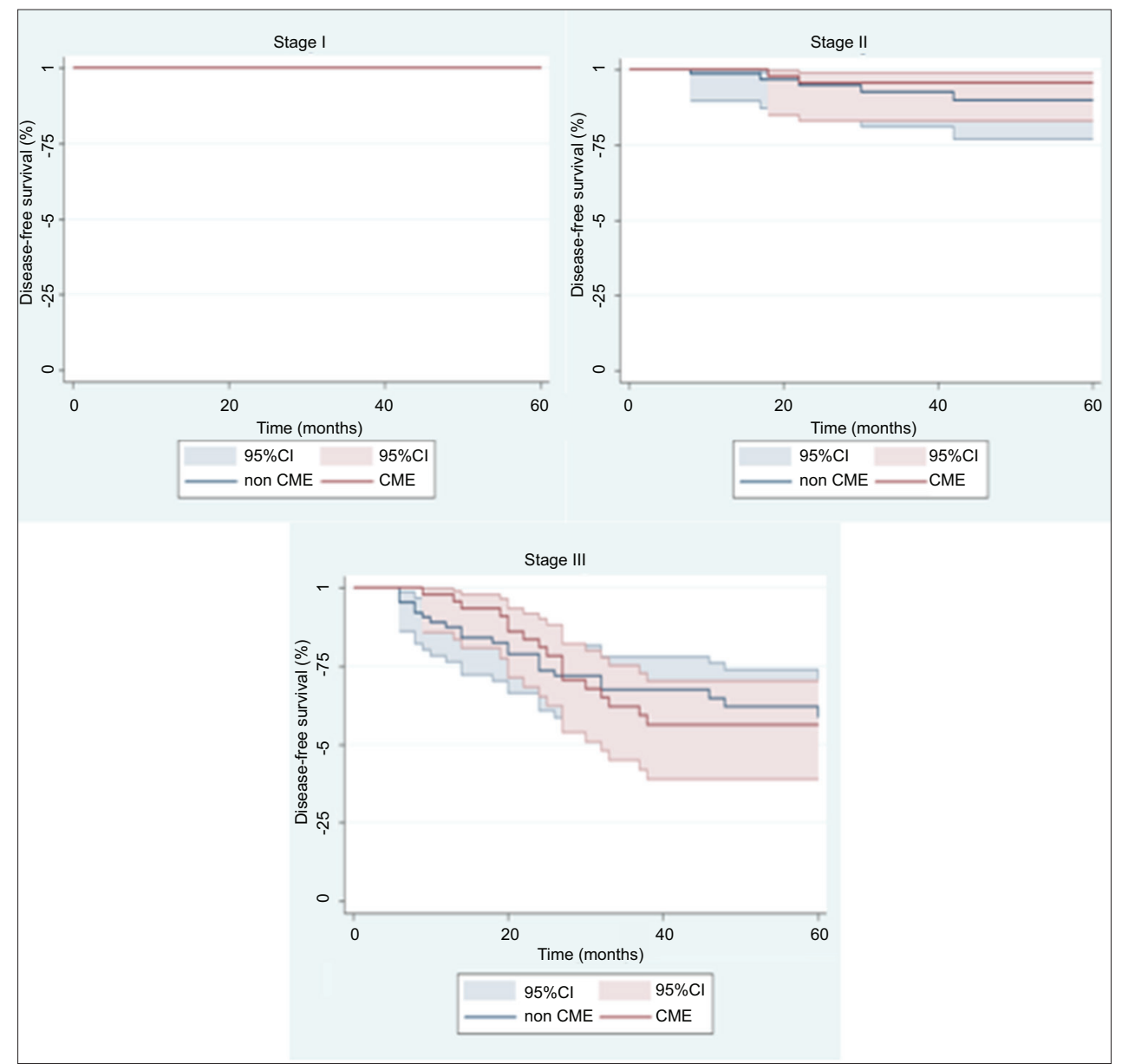

Figure 2 Disease-free survival time between treatment groups, stratified by stage CME, complete mesocolic excision; CI, confidence interval

recent report, by Merkel et al, identified the implementation of CME surgery as one of the most important factors that significantly affected the improvement in disease-free survival and overall survival rates [20]. This could be attributed to several reasons: i) most patients with stage III disease had adjuvant chemotherapy administered, which may affect lymph node metastasis and improve recurrence and survival rates, in this way reducing the absolute effect of CME on the oncological outcome of these patients; ii) as lymph nodes in the CVL region are dissected by CME, but not by conventional colectomy, an upstaging from stage II to stage III is expected to occur in the CME group, whereas in conventional colectomy apical lymph nodes may be missed, leading patients to be classified as stage II if lymph nodes in the vicinity of the tumor are skipped by metastasis; iii) if not meticulous, histopathological examination may miss micro-metastases in apical lymph nodes dissected by CME colectomy. In this case patients are classified as having stage I or II disease. In conventional colectomy such micro-metastases are missed, because apical nodes are not removed, and these patients are also classified as stage I or II disease; and iv) differences in follow-up methods and duration may explain differences in oncological outcomes. In this study all patients followed a strict follow-up schedule, as described in the methods section.

There have been major concerns about increased intraoperative and immediate postoperative morbidity and even mortality after CME colectomy with CVL. This has been attributed to the extent of mesocolic resection, particularly in colectomies involving the right and transverse colon, where CVL is carried out in the region of the superior mesenteric vessels, increasing the risk of vascular injury and injury of the sympathetic nerve plexus. However, all series of CME with CVL show very low mortality and acceptable morbidity rates, with limited intraoperative blood loss. Furthermore, two comparative studies $[13,21]$ show no significant differences in morbidity, mortality or anastomotic leak rates between CME and conventional colectomy. In accordance with the current literature, in the present cohort of patients, no difference between the CME and the non-CME group could be identified regarding mortality, intraoperative complications or reoperation rates.

Our study is subject to many methodological limitations that are commonly found among all studies available in the literature concerning the same issue. Although CME 


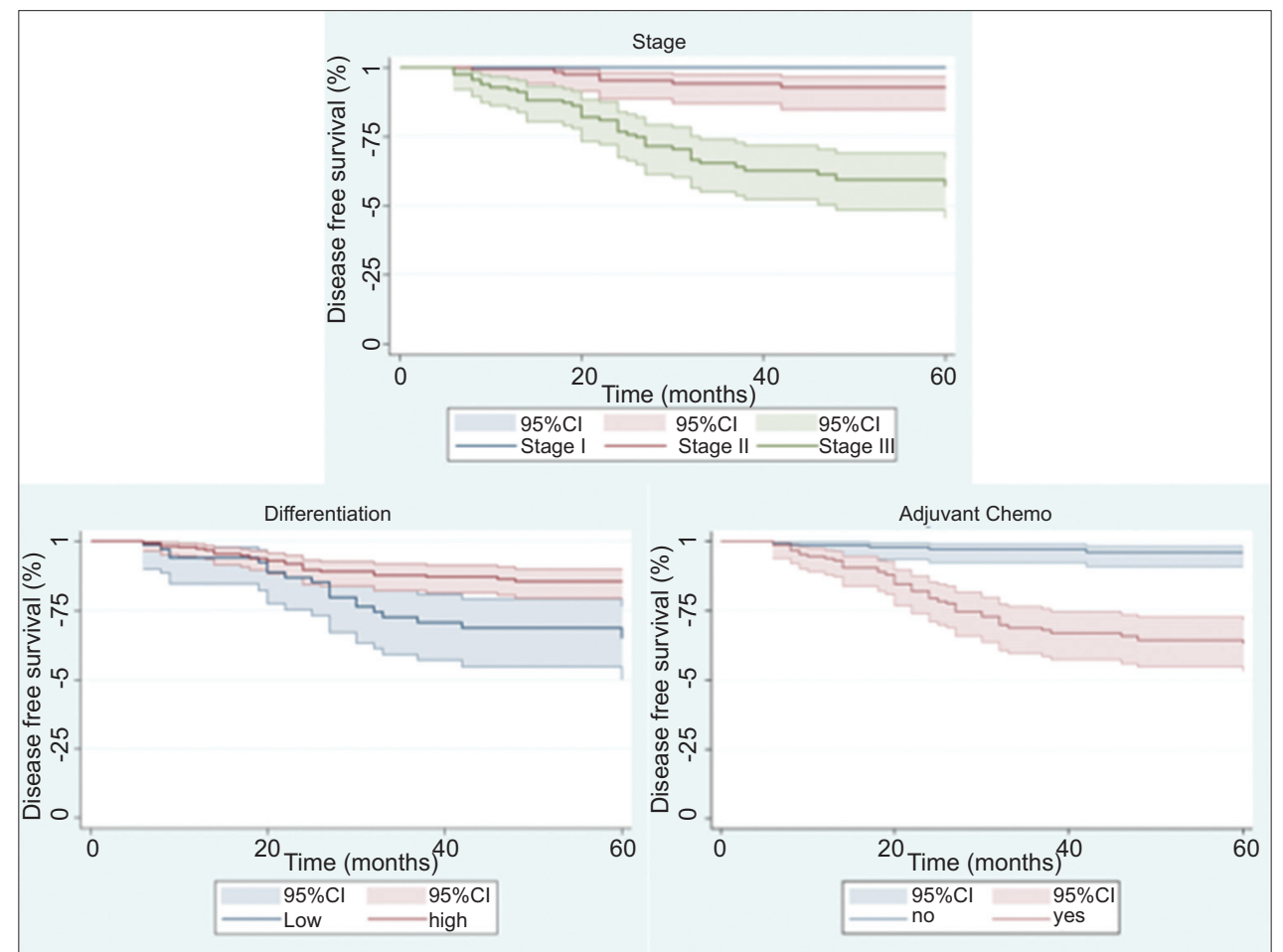

Figure 3 Disease-free survival time by characteristics CI, confidence interval

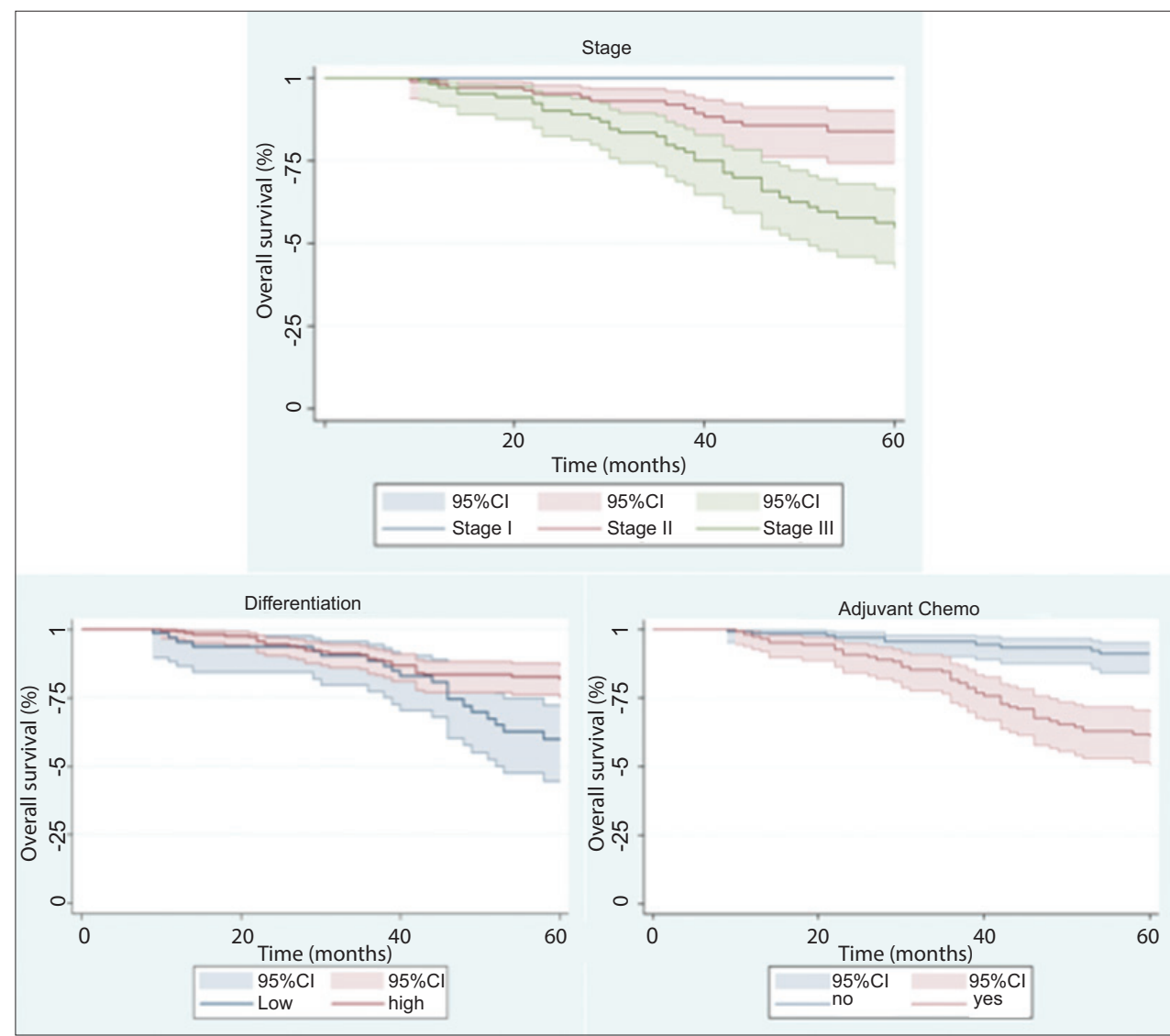

Figure 4 Overall survival time by characteristics

CME, complete mesocolic excision; CI, confidence interval 
Table 4 Multivariate Cox regression model to estimate disease-free and overall survival rates

\begin{tabular}{|c|c|c|c|c|}
\hline \multirow[t]{2}{*}{ Variable } & \multicolumn{2}{|c|}{ Disease-free survival } & \multicolumn{2}{|c|}{ Overall survival } \\
\hline & $\operatorname{HR}(95 \%)$ & P-value & HR $(95 \%)$ & P-value \\
\hline CME & $0.868(0.476-1.584)$ & 0.646 & $0.764(0.405-1.442)$ & 0.406 \\
\hline Differentiation (high) & $0.476(0.261-0.866)$ & 0.015 & $0.441(0.233-0.833)$ & 0.012 \\
\hline Adjuvant chemotherapy & $10.44(4.084-26.689)$ & $<0.001$ & $14.324(4.393-46.709)$ & $<0.001$ \\
\hline Age & $1.036(1.003-1.070)$ & 0.033 & $1.041(1.004-1.078)$ & 0.029 \\
\hline \multicolumn{5}{|l|}{ Stratified by stage } \\
\hline \multicolumn{5}{|l|}{ Stage II } \\
\hline CME & $0.532(0.101-2.798)$ & 0.456 & $0.576(0.105-3.169)$ & 0.526 \\
\hline Differentiation (high) & $0.396(0.086-1.817)$ & 0.234 & $0.706(0.125-3.984)$ & 0.693 \\
\hline Adjuvant chemotherapy & $2.858(0.632-12.921)$ & 0.173 & $4.268(0.773-23.562)$ & 0.096 \\
\hline Age & $1.028(0.952-1.110)$ & 0.483 & $1.059(0.965-1.161)$ & 0.227 \\
\hline \multicolumn{5}{|l|}{ Stage III } \\
\hline CME & $0.979(0.510-1.879)$ & 0.950 & $0.830(0.145-1.659)$ & 0.598 \\
\hline Differentiation (high) & $0.515(0.216-1.015)$ & 0.050 & $0.457(0.222-0.940)$ & 0.033 \\
\hline Adjuvant chemotherapy & $3.606(0.833-15.617)$ & 0.086 & $4.561(0.606-34.335)$ & 0.141 \\
\hline Age & $1.036(0.996-1.079)$ & 0.081 & $1.042(0.997-1.088)$ & 0.067 \\
\hline
\end{tabular}

HR, hazard ratio; CME, complete mesocolic excision

with CVL is a very well standardized operation for tumors located in the cecum, ascending, descending, sigmoid and rectosigmoid colon, the oncological radicality needed for tumors located along the transverse colon, from the hepatic to the splenic flexure, is still an open matter and remains highly controversial, particularly in terms of the extent of lymphadenectomy. At present, in the literature there are a few studies including series of patients with transverse colon tumors who underwent CME with CVL, by either open or laparoscopic approach, but do not report on transverse colon cancers outcomes separately. In one study [22] there is a considerable incidence of metastases of the extramesocolic lymph node stations, which cannot be underestimated and argues in favor of radical lymph node excision in these regions. Unfortunately, in this study the number of true transverse colon adenocarcinomas is also very limited and the outcomes are not presented separately, but at least the cases are equally distributed between the two groups.

A subgroup analysis on the basis of tumor location is therefore mandatory when reporting outcomes for CME plus CVL. Right-sided and transverse colon cancers constitute a different group of tumors, not only because of different biological behavior but also because colectomy in terms of CME differs hugely from the conventional. In this sense, tumor location along the colon is a significant confounder when all cases are being analyzed together. From this viewpoint, CME with CVL is expected to have a greater oncological impact on right and transverse colon cancers. Furthermore, although conventional colectomy for right-sided colonic tumors is even less standardized, conventional left-sided resections are closer to and sometimes coincide with the concept of CME with CVL, probably because they are less technically demanding,

\section{Summary Box}

\section{What is already known:}

- Complete mesocolic excision (CME) results in better quality specimens

- CME leads to increased disease-free survival

- CME is well standardized

\section{What the new findings are:}

- CME leads to increased lymph node harvesting

- CME improves overall survival in tumors with high differentiation

- CME does not increase postoperative complications

which renders them more feasible. It is easily conceivable that probably one or even two centimeters of difference in the CVL between the groups may be not enough to highlight an anticipated statistical difference.

In conclusion, there is strong evidence from this study that, when CME with CVL is performed, a larger amount of tissue, including a greater number of lymph nodes, is excised compared to conventional colectomy. A statistically significant benefit in overall survival was also observed, an outcome that is subject to several confounders such as age, high differentiation of tumors and adjuvant chemotherapy. CME surgery seems to benefit non-aggressive tumors (high differentiation) as far as both 
disease-free and overall survival rates are concerned. However, this study failed to highlight any true oncological superiority of CME compared with conventional colectomy, mostly because of the aforementioned reasons and methodological limitations. On the other hand, this study also demonstrates that CME surgery is a safe, reproducible procedure that is not associated with any increase in severe morbidity and mortality. Large series of patients prospectively undergoing CME with CVL must be accumulated into registries and be compared to conventional surgery cases derived from large archive databases.

\section{Acknowledgment}

The authors would like to deeply thank Drs. Maria Kafousi and Kleio Papaparaskeva for their work and contribution to this work, as they were the two pathologists who examined all resected surgical specimens.

\section{References}

1. Heald RJ, Ryall RD. Recurrence and survival after total mesorectal excision for rectal cancer. Lancet 1986;1:1479-1482.

2. Quirke P, Steele R, Monson J, et al; NCRI Colorectal Cancer Study Group. Effect of the plane of surgery achieved on local recurrence in patients with operable rectal cancer: A prospective study using data from the MRC CR07 and NCIC-CTG CO16 randomised clinical trial. Lancet 2009;373:821-828.

3. Wibe A, Møller B, Norstein J, et al; Norwegian Rectal Cancer Group. A national strategic change in treatment policy for rectal cancerimplementation of total mesorectal excision as routine treatment in Norway. A national audit. Dis Colon Rectum 2002;45:857-866.

4. Hohenberger W, Weber K, Matzel K, Papadopoulos T, Merkel S. Standardized surgery for colonic cancer: complete mesocolic excision and central ligation-technical notes and outcome. Colorectal Dis 2009;11:354-364; discussion 364-365.

5. Gouvas N, Pechlivanides G, Zervakis N, Kafousi M, Xynos E. Complete mesocolic excision in colon cancer surgery: a comparison between open and laparoscopic approach. Colorectal Dis 2012;14:1357-1364.

6. Killeen S, Mannion M, Devaney A, Winter DC. Complete mesocolic resection and extended lymphadenectomy for colon cancer: a systematic review. Colorectal Dis 2014;16:577-594.

7. Kontovounisios C, Kinross J, Tan E, Brown G, Rasheed S, Tekkis P. Complete mesocolic excision in colorectal cancer: a systematic review. Colorectal Dis 2015;17:7-16.

8. West NP, Hohenberger W, Weber K, Perrakis A, Finan PJ,
Quirke P. Complete mesocolic excision with central vascular ligation produces an oncologically superior specimen compared with standard surgery for carcinoma of the colon. J Clin Oncol 2010;28:272-278.

9. West NP, Kennedy RH, Magro T, et al. Morphometric analysis and lymph node yield in laparoscopic complete mesocolic excision performed by supervised trainees. Br J Surg 2014;101:1460-1467.

10. West NP, Kobayashi H, Takahashi K, et al. Understanding optimal colonic cancer surgery: comparison of Japanese D3 resection and European complete mesocolic excision with central vascular ligation. J Clin Oncol 2012;30:1763-1769.

11. West NP, Morris EJ, Rotimi O, Cairns A, Finan PJ, Quirke P. Pathology grading of colon cancer surgical resection and its association with survival: a retrospective observational study. Lancet Oncol 2008;9:857-865.

12. Rosenberg J, Fischer A, Haglind E; Scandinavian Surgical Outcomes Research Group. Current controversies in colorectal surgery: the way to resolve uncertainty and move forward. Colorectal Dis 2012;14:266-269.

13. Galizia G, Lieto E, De Vita F, et al. Is complete mesocolic excision with central vascular ligation safe and effective in the surgical treatment of right-sided colon cancers? A prospective study. Int $J$ Colorectal Dis 2014;29:89-97.

14. Storli KE, Søndenaa K, Furnes B, et al. Short term results of complete (D3) vs. standard (D2) mesenteric excision in colon cancer shows improved outcome of complete mesenteric excision in patients with TNM stages I-II. Tech Coloproctol 2014;18:557-564.

15. Hogan AM, Winter DC. Complete mesocolic excision-a marker of surgical quality? J Gastrointest Surg 2009;13:1889-1891.

16. Hogan AM, Winter DC. Complete mesocolic excision (CME): a "novel" concept? J Surg Oncol 2009;100:182-183.

17. Cho MS, Baek SJ, Hur H, et al. Modified complete mesocolic excision with central vascular ligation for the treatment of rightsided colon cancer: long-term outcomes and prognostic factors. Ann Surg 2015;261:708-715.

18. Gao ZD, Ye YJ, Yang XD, et al. Feasibility of complete mesocolic excision in elderly patients with colon cancer. Zhonghua Wei Chang Wai Ke Za Zhi 2012;15:1023-1026.

19. Guo P, Ye YJ, Jiang KW, et al. Learning curve of complete mesocolic excision for colon cancer. Zhonghua Wei Chang Wai Ke Za Zhi 2012;15:28-31.

20. Merkel S, Weber K, Matzel KE, Agaimy A, Göhl J, Hohenberger W. Prognosis of patients with colonic carcinoma before, during and after implementation of complete mesocolic excision. Br J Surg 2016;103:1220-1229.

21. Bertelsen CA, Neuenschwander AU, Jansen JE, et al; Danish Colorectal Cancer Group. Disease-free survival after complete mesocolic excision compared with conventional colon cancer surgery: a retrospective, population-based study. Lancet Oncol 2015;16:161-168.

22. Perrakis A, Weber K, Merkel S, et al. Lymph node metastasis of carcinomas of transverse colon including flexures. Consideration of the extramesocolic lymph node stations. Int J Colorectal Dis 2014;29:1223-1229. 